\title{
Clinical and Radiographic Findings of Septic Physitis in Foals
}

\author{
David G. Suarez-Fuentes ${ }^{1}$ Dane M. Tatarniuk ${ }^{2}$ \\ ${ }^{1}$ Diagnostic Imaging Service, Department of Small Animal Clinical \\ Sciences, University of Florida College of Veterinary Medicine, \\ Florida, United States \\ 2 Department of Veterinary Clinical Sciences, College of Veterinary \\ Medicine, lowa State University, Ames, lowa, United States
}

\begin{abstract}
Address for correspondence David G. Suarez-Fuentes, DVM, Diagnostic Imaging Service, Department of Small Animal Clinical Sciences, University of Florida College of Veterinary Medicine, 2015 SW 16th Ave, Gainesville, FL 32610, United States (e-mail: dsuarezfuentes@gmail.com).
\end{abstract}

\begin{abstract}
Keywords

- septic

- physitis

- foals

- osteolysis

- lameness

The objective of this review is to detail the clinical and radiographic features of septic physitis in foals. Medical records were evaluated from 2008 to 2018 of cases that had septic physitis based on a combination of clinical and radiographic findings. Ten foals were diagnosed with septic physitis. In 8 of 10 foals, presence of a discrete, focal, irregular radiolucency centred on the physis and extending into the epiphysis and metaphysis was present. In the remaining two foals, subtle irregularity and diffuse radiolucency along the length of the physis was present. Three foals were able to later perform athletic function and two were sound but not in training (mean follow-up $=12$ months). Overall, a distinguishing radiographic feature of septic physitis in foals is the presence of a focal radiolucency centred on the physis which is associated with the epiphysis and metaphysis consistent with osteolysis.
\end{abstract}

\section{Introduction}

Hematogenous spread of bacteria in neonatal foals often occurs secondary to a septic nidus in the body. Pneumonia, colitis and/or an infected umbilicus are the most common infectious pathologies. ${ }^{1-5}$ Often, concurrent failure of passive immune transfer from lack of adequate colostrum intake shortly following birth compounds susceptibility to hematogenous sepsis. ${ }^{1-3,6-8}$ During sepsis, bacteria translocate from the blood stream into the musculoskeletal system, enhanced due to the rich vasculature within the metaphysis, physis, epiphysis and synovium of joints in neonates. Most commonly, septic arthritis develops. ${ }^{1-9}$ In rare cases, infection of open growth plates (septic physitis) can manifest as a primary septic lesion or concurrent with septic arthritis. ${ }^{1,4,8-11}$

Septic physitis in foals has been documented as a musculoskeletal pathology; however, there is a lack of detail regarding characteristic radiographic features in the literature. ${ }^{1,4,6-9,11,12}$ Radiographic examination is the central diagnostic imaging modality used to first identify and/or confirm a diagnosis. ${ }^{1-14}$ Several isolated case reports and one prior retrospective study have been published focusing on clinical presentation and surgical treatment; however, there is a lack of specific description of radiographic characteristics unique to septic physitis. ${ }^{1,4,8,9,11,12}$ Computed tomography (CT) has been investigated as a very effective imaging modality to fully understand the extent of pathology following initial radiographic diagnosis. ${ }^{10} \mathrm{~A}$ prior study comparing radiographic- and $\mathrm{CT}$ images in the diagnosis of osteomyelitis in foals revealed that CT detected lesions that were difficult or impossible to identify on radiographs providing value that $\mathrm{CT}$ should be considered in such cases. ${ }^{13}$ The use of magnetic resonance imaging (MRI) has also been described in the literature in foals with primarily osteomyelitis; however, a low percentage of cases had concurrent extension into the physis. ${ }^{14}$

Radiography often precedes advanced diagnostics in clinical practice, is the mainstay modality in ambulatory practice and advanced imaging (such as CT and MRI) is limited to received

April 10, 2020

accepted

August 24, 2020
DOI https://doi.org/

$10.1055 / \mathrm{s}-0040-1717103$. ISSN 2625-2325. (c) 2020 Georg Thieme Verlag KG
Stuttgart · New York

License terms

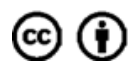


referral centres. Therefore, the objective of this study is to establish common radiographic features of septic physitis based on a case series.

\section{Methods}

Electronic medical records from a single hospital were evaluated from 2008 to 2018. Owners approved use of medical records for research investigation at time of admission. Medical records were searched for 'physitis', 'septic physitis' or 'infected growth plate'. Cases were then analysed to determine whether a clinical and/or radiographic diagnosis of septic physitis was made. Inclusion criteria included (1) radiographic examination performed and a clinical diagnosis of septic physitis based on positive culture from aspiration of the physis or physeal biopsy, septic joint adjacent to the affected physis, umbilicus content culture/biopsy during resection or blood analysis; and/or (2) clinical signs characteristic of local infection (heat, pain, swelling associated with the physis), signs of co-morbidity (fever, failure of passive transfer) and/or clinical pathology consistent with septic arthritis from synovial fluid adjacent to the affected physis. Exclusion criteria included (1) a diagnosis of aseptic physitis by the attending veterinarian or (2) lack of radiographs available for interpretation. All authors evaluated all medical records and formulated agreeance on inclusion or exclusion of individual cases. All authors were involved in data collection, analysis and formulated collective agreeance.

Clinical parameters recorded included signalment (age at presentation, gender, breed), primary complaint, anatomical location, bacteriology results from aspirates or biopsy of the physis, concurrent septic joint(s), blood culture and/or infected umbilicus, presence of concurrent synovial sepsis adjacent or distant from affected physis, clinical pathological abnormalities on complete blood analysis, established aetiology, medical treatment (including type of antimicrobial and route), surgical treatment pursued, short-term patient outcome (defined as survival to discharge) and long-term patient outcome (defined as sound during veterinary examination at $>3$ months following treatment).

Each affected region was evaluated using digital radiography (Sound, Carlsbad, California, United States). The radiographic setting was determined depending on the location of the affected limb and based on manufacturer recommendations. For the carpal joints, proximal interphalangeal joints and metacarpophalangeal/metatarsophalangeal joints, the setting was $70 \mathrm{kVp}, 1.5 \mathrm{mAs}$ and 0.1 seconds. For the stifle and tarsal joints, the setting was $75 \mathrm{kVp}, 1.8 \mathrm{mAs}$ and 0.1 seconds. In all affected regions, dorsopalmar or dorsoplantar/craniocaudal and lateromedial projections were obtained. Radiographic studies were interpreted by multiple different unblinded American College of Veterinary Radiology radiologists at time of patient examination and had an associated final radiology report available within the medical record for recruitment of data.

Radiographic features investigated included documentation of physeal location and limb or limbs affected. Description of the shape, margination and opacification of radiographic abnormalities present in the physis were recorded. To improve objective definition of size and shape, measurements of any lucent regions were obtained in the frontal (lateral to medial view) planes on lateromedial projections and sagittal (dorsal to palmar/plantar, or cranial to caudal view) planes on dorsopalmar or dorsoplantar/ craniocaudal projections. The maximal total physeal abnormality in relation to the width of the physis within the same radiographic plane was used for grading and described in percentage (\%) of the normal total width of the physis. Grade 1 was assigned to cases that had $<33 \%$ of the physis affected, grade 2,33 to $66 \%$ and grade $3>66 \%$. If repeat radiographs were available, serial changes (progression/stasis/regression) in the radiographic appearance of the physis were documented along with duration of days between serial images. Objective data were analysed using descriptive statistics via commercial software (MS Excel, Microsoft Corporation, Redmond, Washington, United States).

\section{Results}

Ten foals met inclusion criteria and were included in the study. The mean age of foals was 52 days (range: 14-182 days). There were six colts and four fillies. Breeds included Thoroughbred $(n=4)$, Quarter Horse $(n=2)$, Standardbred $(n=1)$, Arabian $(n=1)$, Lusitano $(n=1)$ and Pony of the Americas $(n=1)$. All of the Thoroughbred, Quarter Horse and Standardbred foals were bred intended for racing. In nine foals, the presenting complaint was lameness and in one foal generalized lethargy.

Anatomical locations of septic physitis included the distal tibia (4), distal radius (1), proximal first phalanx (1), distal third metatarsus (1), proximal middle phalanx (1), distal femur (1) and proximal tibia (1) (-Fig. 1). Clinically, all infected physes had evidence of subcutaneous oedema, heat and pain to palpation surrounding the affected physis consistent with concurrent cellulitis. In 7 of 10 foals, the lameness was graded as $4 / 5$ (lame at the walk) and in 3 of 10 foals a non-weight-bearing lameness as present. ${ }^{15}$

An attempt to culture the physeal tissue was performed in all ten cases via aspiration or biopsy of bone during surgical debridement. A positive culture result was obtained in 5/10 of cases. Bacteria isolated included Salmonella enterica (1), Klebsiella oxytoca (1), Streptococcus zooepidemicus (2) and mixed growth of Streptococcus dysgalactiae and Escherichia coli (1).

All 10 foals had documentation of systemic co-morbidity. Presence of a fever (defined as $>102.0 \mathrm{~F}^{\circ}$ ) was noted at some point during hospitalization in all 10 cases. Systemic bloodwork was performed in 8 of 10 foals and identified an abnormal total while blood cell count in four of eight cases with a mean count of $13.97 \times 10^{3}$ cells/ $\mu \mathrm{L}$ (range: $11.97-$ $14.6 \times 10^{3}$ cells $\left./ \mu \mathrm{L}\right)$. Fibrinogen concentration was elevated in all eight cases (mean: $850 \mathrm{mg} / \mathrm{dL}$, range: $600-1100 \mathrm{mg} / \mathrm{dL}$ ).

Concurrent septic arthritis of a synovial joint was present in 8 of 10 foals. In seven of eight foals, septic arthritis was located in the joint most closely associated with the septic physis. In one case, the septic arthritis was in the same limb 


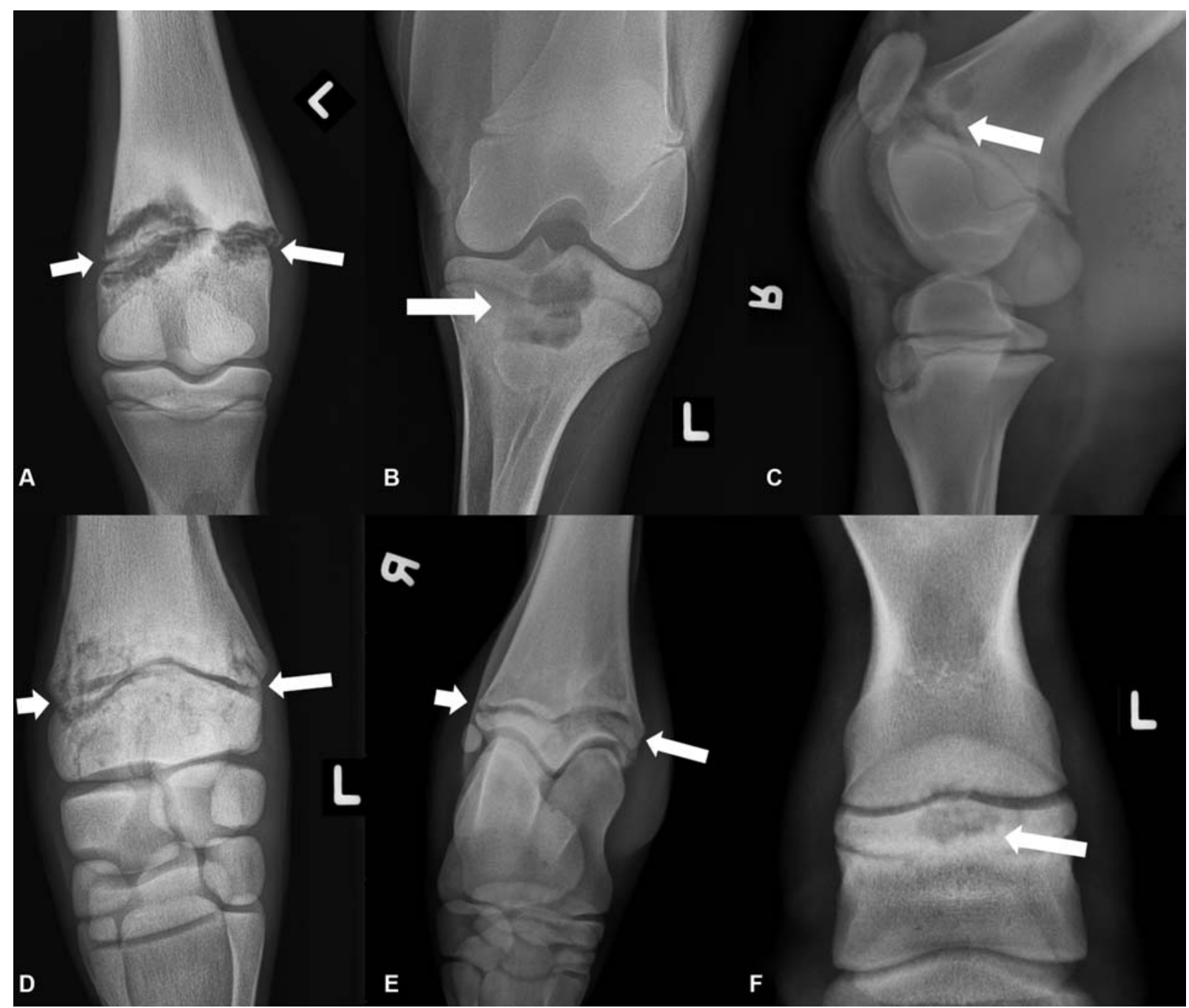

Fig. 1 Radiographic examples of septic physitis in foals in varying anatomical physes with a brief description of the physeal lesions (arrows are pointing the abnormal lesions). (A) Dorsoplantar projection, distal third metatarsus (severe extensive lysis throughout the physis that extends into the metaphysis and epiphysis), 1-month-old Thoroughbred colt; (B) craniocaudal projection, proximal tibia (severe focal lysis centred on the mid physis that extends into the metaphysis and physes), 1-month-old Pony of America filly; (C) lateromedial projection, distal femur (variable mild to moderate focal lysis in the cranial physis that extends into the metaphysis and epiphysis), 3-week-old Thoroughbred colt; (D) dorsopalmar projection, distal radius (variable mild to moderate illdefined lysis throughout the physis that extends into the metaphysis and epiphysis), 2-week-old Thoroughbred filly; (E) dorsoplantar projection, distal tibia (variable mild lysis throughout the physis, worse medially, that extends into the metaphysis and epiphysis), 2-week-old Quarter Horse colt; (F) dorsopalmar projection, proximal middle phalanx (moderate focal lysis centred region of the mid physis that extends into the metaphysis and epiphysis), 3-month-old Thoroughbred colt.

but distant from the region of septic physitis. Synovial fluid obtained from the affected synovial structures was serosanguineous and/or turbid in all affected cases. Culture of synovial fluid was submitted in four of eight foals, with positive growth ascertained in one case (Streptococcus zooepidemicus). Non-musculoskeletal systemic co-morbidities were present in 4 of 10 foals, with two having clinical symptoms of colitis/diarrhoea, one having pneumonia and other having an umbilical abscess confirmed on ultrasonography and resected at surgery.

Overall, in 8 of 10 foals, the septic physis had a focal, irregularly marginated radiolucent region centred within the physis, involving both epiphysis and metaphysis (-Fig. 1). In the remaining two foals, subtle irregularity and less pronounced radiolucency extended diffusely along the length of the physis and into the metaphysis and epiphysis. When evaluating the extent of radiolucency relative to the width of the physis, in the frontal (lateral to medial) plane, the median radiolucent area was 53.4\% (31.2 out of $63.2 \mathrm{~mm}$; range: $15-45 \mathrm{~mm}$ ) of the physeal distance. In the sagittal (dorsal to palmar/plantar or cranial to caudal) plane, the median radiolucent area was $41.0 \%$ (28.8 out of $73 \mathrm{~mm}$; range: $18-53 \mathrm{~mm}$ ) of the physeal width. When measuring the maximal diameter of the radiolucent area in a proximal to distal orientation, the median diameter was $22.8 \mathrm{~mm}$ (range: $3-55 \mathrm{~mm}$ ). Radiographic grading of the physes included grade 1: $1(10 \%)$ case, grade 2: $6(60 \%)$ cases and grade 3: $3(30 \%)$ cases.

Repeat radiographs were performed in 6 of 10 cases. Progression of lesion (enlargement in radiolucent abnormality in physis) was present in one foal, stasis of the radiolucent area was present in four of six foals, and regression of the 


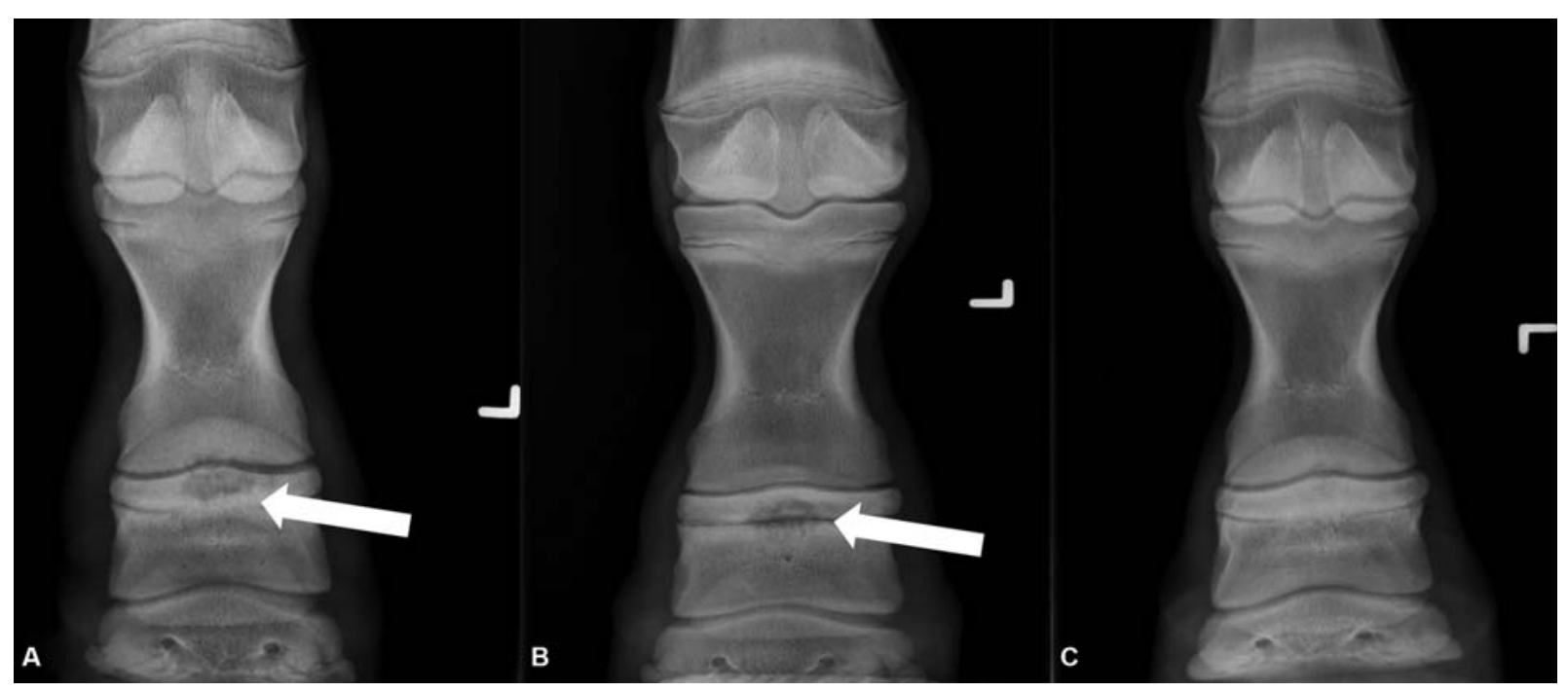

Fig. 2 Dorsopalmar projections of the left front middle phalanx of a 3-month-old Thoroughbred colt with radiographic improvement following medical treatment of septic physitis in the proximal physis of the middle phalanx, left forelimb (arrow is pointing the abnormal lesion). (A) Day 0 (moderate lysis centred in the mid physis that extends into the metaphysis and epiphysis); (B) day 6 (improvement of the described lysis of the physis); (C) day 21 (resolution of the described lysis in the physis).

lesion (reduction in radiolucent abnormality extending into the epiphysis and metaphysis) was present in one foal (-Fig. 2). The mean duration to repeat radiographs was 8 days (range: $4-21$ days).

All septic physitis cases received systemic antimicrobial therapy, often in combination. Antibiotic medications included ceftiofur $(n=3 ; 25 \mathrm{mg} / \mathrm{kg}$ intravenously [IV] twice daily [BID]), gentamicin $(n=3 ; 6.6 \mathrm{mg} / \mathrm{kg}$ IV once daily [SID]), amikacin ( $n=2 ; 2.2 \mathrm{mg} / \mathrm{kg}$ IV SID), penicillin $(n=2$; $22,000 \mathrm{iu} / \mathrm{kg}$, intramuscular or IV, BID or QID respectively), chloramphenicol $(n=1 ; 50 \mathrm{mg} / \mathrm{kg}$ by mouth TID), marbofloxacin ( $n=1 ; 5 \mathrm{mg} / \mathrm{kg}$ IV SID) and/or oral rifampin $(n=1$; $5 \mathrm{mg} / \mathrm{kg}$ by mouth BID). Treatment was augmented with surgical debridement of the affected physis under general anaesthesia in 4 of 10 cases. In one case, gentamicin infused calcium sulphate beads were deposited into the osteolytic area of the physis intra-operatively to provide local antimicrobial support and act as an osteoconductive graft. Intravenous regional limb perfusion was performed in 6 of 10 cases, utilizing amikacin or gentamicin.

Six of 10 horses were discharged from the hospital with improvement in lameness (short-term survival) and 4 of 10 foals were euthanatized during active treatment due to inability to resolve septic physitis and/or systemic co-morbidities. Of the six discharged, three were in active training at 1 -year post-discharge, two were sound but not in work and one foal was lost to follow-up. Of the four foals euthanatized, all four cases had concurrent septic arthritis as a co-morbidity. Additionally, one had a concurrent infected umbilical abscess.

\section{Discussion}

The gold standard for diagnosing septic physitis and/or septic arthritis in foals is a positive microbial culture directly from the affected physis and/or adjacent infected synovial struc- tures. ${ }^{1,2,5}$ In this study, all cases had a microbial culture from the infected physis attempted; however, only 5 of 10 cases yielded positive bacterial growth. This diagnostic challenge is well established in the literature. ${ }^{2,5,16,17}$ It is common for false negative bacteriology following culture of septic joints and osteomyelitis. Similarly, septic physitis is also a difficult pathology to obtain positive identification of infecting bacteria following aspiration or biopsy culture attempts. To maintain confidence that all cases in this study were truly categorized as septic physitis, the authors relied upon culture or cytology from the adjacent infected synovial structure and evidence of infectious systemic co-morbidities consistent with generalized sepsis as support in the remaining cases wherein no positive growth was obtained from the physis itself. Although not definitive, detecting the classical radiographic abnormalities strongly implicates an infectious process underlying the radiographic abnormalities seen.

The most common radiographic feature of septic physitis identified in this dataset is a focal, irregularly marginated radiolucency centred on an open physis, with extension into both the metaphyseal and epiphyseal bone. This radiographic appearance is likely a result of focal lysis that develops due to active infection; however, histopathological evaluation would be necessary to confirm this conclusion. Bacterial infection surrounding and within bone induces widespread inflammation and recruitment of osteoclastic and collagenase activity, leading to local lysis. ${ }^{5-7,11,12}$ It has also been reported that necrotic growth cartilage, secondary to infection, may not ossify correctly and could form areas of retained cartilage that can protrude to the epiphysis and/ or metaphysis which can appear as radiolucent areas on radiographs when compared with adjacent areas with normal ossification. ${ }^{18}$

Aseptic physitis is a non-infectious, inflammatory condition of the physes. ${ }^{11,12}$ Most often observed in foals, weanlings and yearlings, the condition is associated with high- 
energy nutrition and rapid phase of bone growth. ${ }^{5,11}$ In aseptic physitis, a more diffuse, irregular margination of the physis is apparent without the development of focal osteolysis. $^{12}$ It is important to note not all septic physitis cases present typical radiographic changes; two cases identified in our study lacked a focal lucency and more closely resembled the appearance of aseptic physitis. For these two cases noted, concurrent clinical symptoms of septic physitis and positive bacterial culture were obtained to help classify them as septic versus aseptic. One potential explanation for the less classic radiographic appearance is that there were cases of septic physitis that were caught earlier in disease progression. It is well established that changes to bone density on radiographs can be delayed before being radiographically apparent. ${ }^{12}$ These two cases highlight an important point that a lack of a characteristic appearance of septic physitis on imaging, differentiation between septic versus aseptic physitis may be difficult and misclassification could occur.

Outcome following treatment of septic physitis beyond individual case reports and case series is limited to one retrospective report in which all foals received both medical and surgical treatment. ${ }^{1}$ In this report, 15 of 17 treated foals with long-term follow-up were successfully treated. ${ }^{1}$ This prognosis is moderately improved compared with the longterm outcome identified in our dataset. Potentially, pursing surgical curettage of the physis more often could improve case survival. This group of affected foals had a higher prevalence of comorbidities (i.e. umbilical abscess, pneumonia, diarrhoea) that likely resulted in a lower prognosis despite aggressive treatment of the septic physis. It is also unknown whether financial limitations influenced inability to achieve clinical resolution of septic physitis in our study. Overall, this study maintains consistency with the literature that aggressive treatment of septic physitis can result in a fair to favourable prognosis. A larger cohort of clinical cases is urgently needed to draw firm conclusions regarding medical treatment versus combined medical and surgical treatment of septic physitis, and subsequent influence on prognosis for survival and future athleticism.

To best characterize septic physitis in clinical cases, CT should be considered. CT has been previously described as a post-mortem diagnostic to better delineate the extent of pathology seen on radiographs. ${ }^{10}$ The authors are currently recommending $\mathrm{CT}$, performed under sedated restraint, in ongoing clinical cases to better evaluate and prognosticate severity of septic physitis to clients at the onset of diagnosis.

Limitations of this study include the retrospective nature of the investigation. The inability to obtain a positive culture from the affected physis itself in selected cases limits the absolute confirmation of septic physitis as being present. The small sample size precludes statistical analysis. Ideally, comparing size of radiolucency with outcome would be of clinical interest to identify any underlying correlations. Radiologists were not blinded to the clinical findings of the patient prior to interpreting radiographic images.

In conclusion, a characteristic radiographic feature of septic physitis is a radiolucency centred on the affected physis that is focal and irregular in margination. The size of the radiolucency is variable, presumably due to differing degrees of osteolysis, bacterial pathogenicity, chronicity of infection and differing physeal regions. In this retrospective report, anatomical locations affected are highly variable, with a slight predisposition toward the distal tibial physis in this retrospective report. Careful attention to common radiographic features of affected physes will facilitate equine veterinarians establishing a correct and timely diagnosis.

\section{Authors' Contributions}

D.G.S.-F. and D.M.T. conceptualized and designed the study, and acquired, analysed and interpreted the data. All authors drafted, revised and approved the submitted manuscript.

\section{Note}

This manuscript has been presented in oral abstract form at the 2019 Veterinary Orthopedic Society Scientific Conference, Snowbird, Utah, United States, February 11, 2019, and the 2018 ACVR/IVRA Scientific Conference, Fort Worth, Texas, United States, October 14-19, 2018.

\section{Conflict of Interest}

None declared.

\section{References}

1 Hall MS, Pollock PJ, Russell T. Surgical treatment of septic physitis in 17 foals. Aust Vet J 2012;90(12):479-484

2 Firth EC. Current concepts of infectious polyarthritis in foals. Equine Vet J 1983;15(01):5-9

3 Reig Codina L, Werre SR, Brown JA. Short-term outcome and risk factors for post-operative complications following umbilical resection in 82 foals (2004-2016). Equine Vet J 2019;51(03): 323-328

4 Kettner NU, Parker JE, Watrous BJ. Intraosseous regional perfusion for treatment of septic physitis in a two-week-old foal. J Am Vet Med Assoc 2003;222(03):346-350, 316

5 Richardson D, Ahern B. Synovial and osseous infections. In: Auer JA, Stick JA, eds. Equine Surgery. 4th edition Philadelphia: Saunders; 2012:1189-1199

6 Neil KM, Axon JE, Begg AP, et al. Retrospective study of 108 foals with septic osteomyelitis. Aust Vet J 2010;88(1-2):4-12

7 Hardy J. Etiology, diagnosis, and treatment of septic arthritis, osteitis, and osteomyelitis in foals. Clin Tech Equine Pract 2006; 5:309-317

8 Baird AN, Taylor JR, Watkins JP. Debridement of septic physeal lesions in 3 foals. Cornell Vet 1990;80(01):85-95

9 Parviainen AK. Septic physitis in a foal. Compend Contin Educ Pract Vet 2002;24:73-74

10 Munsterman AS, Alexander K, Samii VF, Latimer FG. Computed tomography in the diagnosis of septic physitis in two foals. Equine Vet Educ 2007;19:200-206

11 Baxter GM. Epiphysitis/physitis/physeal dysplasia. In: Baxter GM, ed. Adams \& Stashak's Lameness in Horses. 6th edition Ames, IA: Blackwell Publishing; 2011:1132-1135

12 Thrall D. Textbook of Veterinary Diagnostic Radiology. 7th edition St Louis: Elsevier; 2018:385-386

13 Lean NR, Perkins NR, Ahern BJ. Comparison of conventional radiography and computed tomography as aids in the diagnosis of osteomyelitis in 11 foals. Aust Vet J 2018;96(07):257-261 
14 Gaschen L, LeRoux A, Trichel J et al. Magnetic resonance imaging in foals with infectious arthritis. Vet Radiol Ultrasound 2011; 52:627-633

15 Anon. Definition and classification of lameness. Guide for Veterinary Service and Judging of Equestrian Events 1st edition Lexington, Kentucky: American Association of Equine Practitioners; 1991:19

16 Madison JB, Sommer M, Spencer PA. Relations among synovial membrane histopathologic findings, synovial fluid cytologic find- ings, and bacterial culture results in horses with suspected infectious arthritis: 64 cases (1979-1987). J Am Vet Med Assoc 1991;198(09):1655-1661

17 Schneider RK, Bramlage LR, Moore RM, Mecklenburg LM, Kohn CW, Gabel AA. A retrospective study of 192 horses affected with septic arthritis/tenosynovitis. Equine Vet J 1992;24(06):436-442

18 Wormstrand B, Østevik L, Ekman S, Olstad K. Septic arthritis/ osteomyelitis may lead to osteochondrosis-like lesions in foals. Vet Pathol 2018;55(05):693-702 